Journal of Bioinformatics and Computational Biology

Vol. 14, No. 4 (2016) 1650015 (22 pages)

(C) The Author(s)

DOI: $10.1142 / S 0219720016500153$

\title{
Gene-set activity toolbox (GAT): A platform for microarray-based cancer diagnosis using an integrative gene-set analysis approach
}

\author{
Worrawat Engchuan \\ Data and Knowledge Engineering Laboratory \\ School of Information Technology \\ King Mongkut's University of Technology Thonburi \\ Bangkok, Thailand \\ worrawat.eng@sit.kmutt.ac.th \\ Asawin Meechai \\ Department of Chemical Engineering \\ Faculty of Engineering \\ King Mongkut's University of Technology Thonburi \\ Bangkok, Thailand \\ asawin.mee@kmutt.ac.th \\ Sissades Tongsima \\ Biostatistics and Informatics Laboratory \\ Genome Technology Research Unit \\ National Center for Genetic Engineering and Biotechnology \\ sissades@biotec.or.th \\ Narumol Doungpan \\ Biological Engineering Program \\ Faculty of Engineering \\ King Mongkut's University of Technology Thonburi \\ Bangkok, Thailand \\ narumol.57@mail.kmutt.ac.th \\ Jonathan H. Chan \\ Data and Knowledge Engineering Laboratory \\ School of Information Technology \\ King Mongkut's University of Technology Thonburi \\ Bangkok, Thailand \\ jonathan@sit.kmutt.ac.th
}

Received 17 November 2014

Revised 4 February 2015

Accepted 29 February 2016

Published 21 April 2016

This is an Open Access article published by World Scientific Publishing Company. It is distributed under the terms of the Creative Commons Attribution 4.0 (CC-BY) License. Further distribution of this work is permitted, provided the original work is properly cited. 


\title{
W. Engchuan et al.
}

\begin{abstract}
Cancer is a complex disease that cannot be diagnosed reliably using only single gene expression analysis. Using gene-set analysis on high throughput gene expression profiling controlled by various environmental factors is a commonly adopted technique used by the cancer research community. This work develops a comprehensive gene expression analysis tool (gene-set activity toolbox: (GAT)) that is implemented with data retriever, traditional data pre-processing, several gene-set analysis methods, network visualization and data mining tools. The gene-set analysis methods are used to identify subsets of phenotype-relevant genes that will be used to build a classification model. To evaluate GAT performance, we performed a cross-dataset validation study on three common cancers namely colorectal, breast and lung cancers. The results show that GAT can be used to build a reasonable disease diagnostic model and the predicted markers have biological relevance. GAT can be accessed from http://gat.sit.kmutt.ac.th where GAT's java library for gene-set analysis, simple classification and a database with three cancer benchmark datasets can be downloaded.
\end{abstract}

Keywords: Microarray; gene expression analysis; gene-set; classification; feature selection; breast cancer; lung cancer; colorectal cancer.

\section{Introduction}

Despite significant advances in early cancer detection, treatment, and prevention, we still observe high incidence and mortality rates for all types of cancers, ${ }^{1}$ with even greater risks as we get older. ${ }^{2}$ With the advent of an aging society, accurate cancer diagnosis is needed in order for early and effective detection of cancer conditions and to reduce the underlying mortality rate. ${ }^{3-5}$ Large numbers of microarray datasets have continually been generated and deposited into public databases. These datasets enable researchers to test many novel bioinformatics algorithms. ${ }^{6-9}$ However, finding cancer markers to effectively classify the disease is a very challenging problem. Various studies have questioned the reliability of cancer classifiers derived from gene expression analysis. ${ }^{8,10,11}$ To address this, the integrative gene-set analysis was introduced as it can capture a more comprehensive picture of changes in the biological process of complex diseases such as cancer. ${ }^{12,13}$ Gene-set analysis is commonly practiced to identify gene-set markers for classifying complex disease. ${ }^{4,14}$ Gene-set enrichment analysis (GSEA) is a well-known gene-set analysis method used to identify gene-sets associated with a given phenotype. ${ }^{15}$ Along with the development of GSEA, the molecular signature database (MSigDB) was constructed to offer a huge public domain gene-set collections. ${ }^{15}$ Currently, several gene-set analysis tools use gene-set activity to classify diseases using gene expression profiles. ${ }^{5,16,17}$ Based on the variation in gene expression levels, these disease classifiers select gene-set markers used in their classification processes. ${ }^{4,5,16-21}$ Lee et $a .^{17}$ proposed the conditionresponsive genes (CORGs)-based method that uses a greedy heuristic to identify CORGs set of each gene-set. The gene expression levels of CORGs set are then summarized to represent an activity level of that gene-set. The performance of the CORGs-based method was evaluated with many cancer datasets. This transformed activity was shown to be robust and provided more discriminative power than the use of gene expression level. Subsequent works focus on using a subset of phenotypecorrelated genes (PCOGs) to uniquely identify pathways related to breast 
cancer. ${ }^{18,19}$ The use of negatively correlated feature sets-based method (NCFS- $i$ ) for identifying PCOGs in each pathway was shown to improve the robustness and increase the discriminative power of disease detection than previous studies. Nevertheless, the NCFS- $i$ and CORGs-based gene-set activity transformation methods are limited to binary-class datasets only. To cope with this limitation, the analysisof-variance or ANOVA-based feature set method (AFS) was proposed to handle gene-set activity in multi-class classification problems. ${ }^{20}$ These gene-set selection techniques may also be used to select important features, i.e. phenotype-correlated genes, during the PCOGs/CORGs identification process. Thus, the selected gene subsets can be used to efficiently classify phenotypes.

Furthermore, the structure of gene-set (gene network or gene-gene interactions) can also be considered to improve the gene-set analysis. We have developed a genenetwork-based feature set (GNFS) method (unpublished work) ${ }^{21}$ that utilizes both gene expression and gene-gene interaction information to identify phenotype-related genes for multi-class problems. The ANOVA test is applied in the GNFS tool to filter out genes with low variation between groups. The genes with $p$-values (lower than a predefined threshold) are mapped to a draft gene network, called scaffold, which is constructed from the gene-gene relationship data. This method deploys greedy search to identify disease-gene sub-networks from the hub genes of each gene-set. Paired fuzzy SNet or PFSNet is another existing network-based analysis. ${ }^{22}$ PFSNet uses majority vote to select a subset of genes based on calculated "fuzzy scores". Then, the whole gene network is separated into sub-networks by mapping those genes onto it. The disease sub-networks are selected by performing a paired $t$-test and permutation test to determine the significance. The idea is to focus on highly expressed genes because they are commonly found across independent datasets.

Although many gene-set analysis methods have been proposed, with even more novel algorithms being developed, using these proposed methods can be limited by either users' coding skill to program those algorithms or having limited computational resources. Hence, we propose an integrated toolbox for gene-set analysis termed gene-set activity toolbox (GAT) that incorporates some of the above mentioned gene-set microarray analysis methods and machine learning tools via java modules implementation with network visualization. In particular, the toolbox offers a gene-set analysis environment where algorithm developers and data analysts can collaborate. Those gene-set analysis algorithms can then be compared while the data analyst can experiment on various analysis methods.

For any gene-set analysis for disease diagnosis, the ultimate goal is to produce high quality results in terms of (i) the classification performance over new batches of data, (ii) the reproducibility of markers selected across datasets and (iii) low falsepositive rates of control experiments. In this study, we focus on the classification performance and the reproducibility of the markers. We demonstrate GAT performance for disease classification by using three common cancers, i.e. lung, colorectal, and breast cancers, ${ }^{23}$ as a case study. The results are then assessed by performing cross-dataset validation and measuring the marker agreements. Finally, we show 


\section{W. Engchuan et al.}

that GAT can readily be used to identify gene subsets for constructing a disease diagnosis model.

The organization of the remaining sections is as follows. Section 2 explains the developed toolbox, followed by details on the datasets used and experimental design of the case study in Sec. 3. Section 4 reports the experimental results of cross-dataset validation. Then Sec. 5 provides an in-depth analysis and discussion of this work. Finally, the last section concludes the findings and suggests some future directions.

\section{Gene-set Activity Toolbox}

An online comprehensive web-based version of GAT is presented in this work. By registering an account with GAT, a user will be able to perform the gene-set analysis with personal login and temporary storage of 2 GB. The core GAT includes many gene-set analysis algorithms including AFS, GNFS, NCFSi and CORGs-based, as well as a simple classification tool and KEGG mapper for classification and biological interpretation purposes. Network visualization with protein-protein interaction (PPI) mapper and PinnacleZ, ${ }^{49}$ a tool for gene sub-network identification, are also included in GAT. GAT can identify the disease-relevant genes from gene-set and transform the gene expression levels to gene-set activity. The overall workflow and features of GAT are shown in Fig. 1.

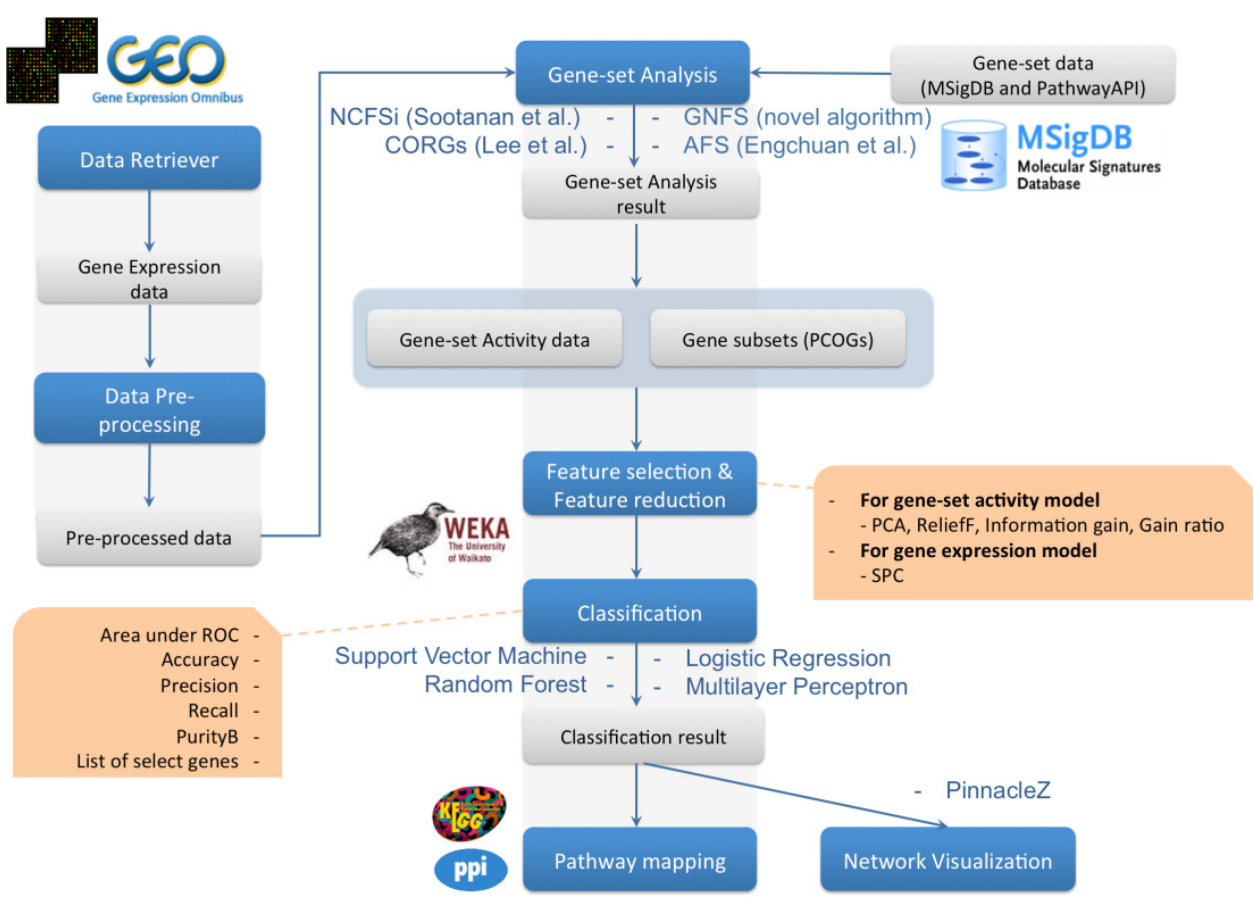

Fig. 1. The workflow and features of the developed GAT. 
First, a microarray dataset can be retrieved from the gene expression omnibus (GEO) datasets ${ }^{26}$ or uploaded by a user. The dataset are then normalized using $z$-score transformation for each gene across all samples as follows:

$$
Z_{i j}=\frac{x_{i j}-\mu_{i}}{\sigma_{i}}
$$

where $x_{i j}$ represents the expression value (raw score) of gene $i$ from sample $j, \mu_{i}$ represents the mean of all samples (population) of gene $i$, and $\sigma_{i}$ represents the standard deviation of gene $i$ in the population.

Second, the gene-set data are collected from MSigDB ${ }^{15}$ and $\mathrm{PathwayAPI}^{27}$ for gene-set integrative analysis purposes. The gene expression levels are mapped to each gene-set. Then the identification of disease-relevant genes and the transformation to gene-set activity are performed. For the construction and validation of a disease diagnosis model, GAT makes use of Waikato environment for knowledge analysis (WEKA) library version $3.7 .10^{28}$ and provides both $k$-fold cross-validation and crossdataset validation. For feature selection and feature reduction, four general methods including principal component analysis (PCA), Relief-F, gain ratio and information gain are available for gene-set activity model, while single pathway classification (SPC) is available for gene expression model. Support vector machine (SVM), multilayer perceptron (MLP), random forest (RF), and logistic regression (LR) are selected as classification algorithms for this release. Presently, GAT requires a user to specify the number of features used to build a classification model (adjustment of classification parameters is not yet available). Classification result is reported as classification performance in terms of accuracy, area under the receiver operating characteristic curve (AUC), precision, recall and PurityB (purity index). PurityB is for assessing the compatibility levels between two datasets, this measurement ranges from 0 to 1 where 0 represents high compatibility level and vice versa. ${ }^{24}$ Furthermore, the selected genes can be used for functional analysis by mapping selected genes to the KEGG pathways ${ }^{29}$ or PPI network. Finally, a network visualization tool is provided along with an implementation of a sub-network identification module based on PinnacleZ. ${ }^{49}$

\section{Demonstration of GAT}

A case study is conducted to demonstrate the key features of GAT. Here, we perform the gene-set analysis using AFS and GNFS methods. A brief description of these two techniques is given below.

\subsection{ANOVA-based feature set}

Engchuan and Chan ${ }^{20}$ proposed AFS to do gene-set activity transformation for multi-class classification problems. The gene-set activity represents the active levels of a particular gene-set. This algorithm uses ANOVA to evaluate the significance of gene or gene-set associated with a disease phenotypic outcome. In each gene-set, gene 


\section{W. Engchuan et al.}

members are sorted in a descending order according to their $F$-values. Genes that are correlated with the given phenotype, termed PCOGs, are identified in a greedy fashion. Initially, the first top gene is assigned to be the first member of a positive group, with the gene's corresponding expression level taken to be the gene-set activity. In the next iteration, another top gene is added to this PCOGs set and the gene-set activity is re-calculated again. Pearson's correlation coefficient is applied to calculate the correlation between this new gene and the first gene. If it has a positive correlation, the new gene will be assigned to the positive group, and the negative group, otherwise. The expression levels of genes in the positive and negative groups are normalized independently as follows (Eq. (2)).

$$
S=\frac{\sum_{i=1}^{n} Z_{i j}}{\sqrt{n}},
$$

where $S$ is a summarized expression level, $\mathrm{Z}_{i j}$ is a $z$-transformed gene expression of gene $i$ from sample $j$ and $n$ is a number of genes in PCOGs of a particular group.

Then, the summarized expression level of the positive group is subtracted by that of the negative group as a new gene-set activity (Eq. (3)).

$$
\text { Gene-set activity }=S_{\text {pos }}-S_{\text {neg }},
$$

where $S_{\text {pos }}$ and $S_{\text {neg }}$ are summarized expression levels from positive and negative groups, respectively. ANOVA is applied to evaluate the significance of the previous gene-set activity and the new one. If the $F$-value of the new gene-set activity is higher than the previous one, the next top gene is added to the PCOG sets; otherwise, the new gene will be removed from PCOGs. These steps are repeated until there is a decrease in the resulting $F$-value, or no more genes can be added to PCOGs.

\subsection{Gene-network-based feature set}

Gene-network-based feature set is a gene subset selection and gene-set activity transformation approach currently in development. ${ }^{21}$ This framework combines gene-set and network data to improve both robustness and biological relevance of selected markers. For each gene-set, the normalized expression levels of the genes are aligned with the gene-set members. ANOVA is used to test the difference in variation among groups of samples. Only genes with ANOVA $p$-value less than a given threshold (default threshold $=0.05$ ) are mapped onto the network scaffold data. We assume that those genes that pass the threshold interact to one another and contribute to disease susceptibility. This step generates draft gene sub-networks (network scaffolds). Hub genes can be identified by considering those top $k$ genes with the highest connectivity (genes with the highest number of edges in neighbor nodes). For each hub gene, the sub-network is constructed by including the neighbor genes using a greedy heuristic. Pearson's correlation coefficient is used to calculate the correlation coefficient between hub and neighbor nodes. If the coefficient is positive, the neighbor node is then placed in the positive group, where hub gene belongs, 
otherwise it is put in the negative group. The gene-set activity is calculated from the subtraction of the cumulative results from positive and negative groups. The discriminative score of gene-set activity calculated using ANOVA and the $F$-value is used as a scoring metric. The neighbor node with higher $F$-value is permanently added to the current sub-network. Termination is reached when no neighbor gene can be added to increase the current $F$-value. Among all hub genes, the sub-network having the highest $F$-value is selected as the representative of the gene-set sub-networks.

\subsection{Gene-set-based analysis as feature selection}

Besides transforming into gene-set activity, GAT can utilize PCOGs obtained from AFS and GNFS as the important features (termed gene-set-based feature selection) for disease classification. An example of gene-set-based feature selection using AFS is described as follows. Let $S_{1}(n)$ be the gene-set having genes $A, B, C, D, E$ as members. PCOGs of $S_{1}(n)$ identified by AFS is represented by $\mathrm{PCOG}_{1}(m)$, containing genes $A, D, E$. Let $\mathrm{PCOG}_{2}(j)$ having genes $U, W, X$ as members represent PCOGs of gene-set $S_{2}(i)$, and $\mathrm{PCOG}_{2}(j)$ is a subset of $S_{2}(i)$. Thus, using PCOGs of gene-sets $S_{1}(n)$ and $S_{2}(i)$ as gene-set-based gene markers, in the classification step, $\mathrm{PCOG}_{1}(m)$ combining with $\mathrm{PCOG}_{2}(j)$ genes will be used as features (genes $A, D, E$, $U, W, X)$.

In our experiment, subsets of PCOGs with at least five genes in the set are selected for building the classification model in AFS. For GNFS, the threshold for ANOVA testing is set to 0.05 and the minimum size of a gene sub-network is set as three genes since five would result in too little number of sub-networks being identified.

\subsection{Microarray datasets and gene-set collections}

This study demonstrates GAT's functionality and usability as well as its performance by using publicly available mRNA expression datasets covering three different disease classification scenarios. Two independent datasets for each scenario are chosen so that cross-dataset validation can be done. The scenarios are: (1) two expression profiles for 30 and 62 breast cancer patients studied by Turashvili et al. ${ }^{30}$ (GSE5764) and Richardson et al. ${ }^{31}$ (GSE7904), respectively; (2) two colorectal cancer expression profiles for 22 and 64 patients studied by Hong et al. ${ }^{32}$ (GSE4107) and Sabates-Bellver et al. ${ }^{33}$ (GSE8671), respectively; 3) and the two multi-class lung cancer datasets (GSE2109 and GSE43580) for 52 and 150 patients provided in the SBV Improver competition. ${ }^{34}$ Each dataset is divided into multiple populations of distinct phenotypes as indicated in the original publication (see Table 1). These datasets can be analyzed readily with their expression levels and can be retrieved from the public database of GEO. ${ }^{26}$

In terms of quality control, the microarray probes with expression level of more than one gene in a platform are discarded in this study to remove potentially ambiguous interpretation of the results. 
Table 1. Microarray datasets used in this study.

\begin{tabular}{|c|c|c|c|c|}
\hline Series ID & Name & Cancer type & Publication & Samples and classes \\
\hline GSE5764 & Breast1 & Breast cancer & Turashvili et al. ${ }^{30}$ & $\begin{array}{c}\text { normal: } 20 \text { samples } \\
\text { tumor: } 10 \text { samples }\end{array}$ \\
\hline GSE7904 & Breast2 & Breast cancer & Richardson et al. ${ }^{31}$ & $\begin{array}{c}\text { normal: } 19 \text { samples } \\
\text { tumor: } 43 \text { samples }\end{array}$ \\
\hline GSE4107 & Colorectal1 & Colorectal cancer & Hong et l. $^{32}$ & $\begin{array}{c}\text { normal: } 10 \text { samples } \\
\text { tumor: } 12 \text { samples }\end{array}$ \\
\hline GSE8671 & Colorectal2 & Colorectal cancer & Sabates-B, et al. ${ }^{33}$ & $\begin{array}{c}\text { normal: } 32 \text { samples } \\
\text { tumor: } 32 \text { samples }\end{array}$ \\
\hline GSE43580 & MCLung1 & Multi-class Lung cancer & Tarca et al. ${ }^{34}$ & $\begin{array}{l}\text { AC stage 1: } 41 \text { samples } \\
\text { AC stage 2: } 36 \text { samples } \\
\text { SCC stage 1: } 34 \text { samples } \\
\text { SCC stage 2: } 39 \text { samples }\end{array}$ \\
\hline GSE2109 & MCLung2 & Multi-class Lung cancer & Tarca et al. ${ }^{34}$ & $\begin{array}{l}\text { AC stage 1: } 17 \text { samples } \\
\text { AC stage 2: } 6 \text { samples } \\
\text { SCC stage 1: } 21 \text { samples } \\
\text { SCC stage 2: } 8 \text { samples }\end{array}$ \\
\hline
\end{tabular}

For the demonstration of GAT, since the gene-set collections from MSigDB do not provide the gene-gene relationship data required by GNFS, the pathway data from PathwayAPI are used instead. PathwayAPI is the collection of pathways from three major pathway databases (KEGG, Ingenuity and Wikipathways). ${ }^{27}$ The pathway data contain 319 gene-sets covering 7467 genes and 60,853 gene-gene relationships.

\subsection{Disease classification}

In this work, we use three well-known classification algorithms: SVM, MLP and RF. SVM is a supervised learning method that analyzes data and recognizes patterns, originally used for classification and regression analysis. ${ }^{35}$ SVM has been widely used as both classification and feature selection in microarray data analysis; it was reported to outperform other classifiers when dealing with multi-class problems. ${ }^{36}$ The neural network classifier, MLP with back-propagation has been commonly applied to classification and diagnosis of cancers based on expression profiles. ${ }^{37}$ The stochastic classifier, RF classifies subjects based on a majority vote of multiple decision trees generated via the bagging technique. Because RF can avoid over-fitting, it has been used in numerous bioinformatics research domains. ${ }^{38,39}$

\subsection{Classification performance evaluation}

The effectiveness of classification using gene-set-based gene markers is evaluated by measuring their classification performance using cross-dataset validation. The crossdataset validation builds a model using a training set and treats the second dataset 
as an independent test set. For the evaluation of the performance of a classifier, AUC is used because it is unbiased towards class-imbalanced issues, and by using oneversus-one strategy, AUC has shown to be well suited for multi-class classification problems. $^{40}$

Like all classifiers, the ones used in GAT are subjected to having classification errors; namely, false positive (type I error) and false negative (type II error). For medical screening purposes, type II errors are more important when compared with type I. The reason is that if there is a misclassification of a patient as a healthy person, then the patient may not receive proper treatment after the diagnostic step. Hence, to measure the model effectiveness, recall is also selected to better represent the performance of the model in medical screening. ${ }^{41}$ So, the final classification performance is reported as both the ROC area and the recall of cross-dataset validation (between datasets).

\subsection{Further analysis of result and visualization}

The significant genes identified from high throughput expression data, e.g. microarray data can be used as a disease biomarker for disease diagnosis. In particular, large numbers of genes may be identified from the analysis, which is not applicable or efficiently used as disease biomarker. To overcome this issue, these genes are further considered in their functional role on the basis of interaction to obtain the genes aggregating the signal for disease state to be considered as disease biomarker. PinnacleZ proposed by Taminau et $a l^{42}$ is a method for network-based biomarker identification using gene expression and PPI network. In their work, PinnacleZ has been used for breast cancer metastasis classification. This algorithm is also included in GAT, which can be used for sub-network identification from both the whole expression dataset and the pre-selected set of genes. GAT is also implemented with network visualization, which benefits the analysis of big data by enhancing user perception. Additionally, visualization helps network interpretation and also depicts how the genes resulting from each algorithm interacts with one another based on PPI data.

\section{Results}

For the demonstration of GAT, we conduct a case study by using AFS and GNFS to analyze six microarray datasets. The existing tool of gene-set-based gene expression analysis with the integration of gene network data, namely Paired Fuzzy SNet or PFSNet, is used as a benchmarking tool in the demonstration. The gene-sets identified by GNFS, PFSNet and AFS with defined parameters are compared. The classification performance is evaluated using cross-dataset validation and reported as AUC and recall. In addition, as in some datasets, large numbers of genes are identified as markers by PFSNet, those genes are used as prior knowledge for disease biomarker identification by PinnacleZ. The reproducibility of selected markers is 
presented in terms of gene-set level and gene level agreements. Finally, the reliability of the results can be assessed using the purity index.

\subsection{Comparison with PFSNet}

In addition to the classification performance, another goal of a robust gene expression analysis approach is the ability to identify consistent markers across different datasets. In other words, markers of a particular disease identified from two independent datasets are expected to have a high level of agreement. However, it is commonly found that the agreement level of markers between two independent studies is quite low. ${ }^{43}$ PFSNet which is a gene-set-based gene expression analysis integrating with gene network data was recently proposed to address this issue. PFSNet identifies disease sub-networks by using a fuzzy concept. The fuzzy score of each gene of each sample is calculated based on its percentile. Score will be determined based on two thresholds ( $\mathrm{t} 1$ and $\mathrm{t} 2$ ). If percentile of a gene is higher than $\mathrm{t} 1$, the score will be assigned as 1 , while it will be 0 if the percentile is lower than $t 2$. For those genes having percentile between $\mathrm{t} 1$ and $\mathrm{t} 2$ will have score ranging from 0 to 1 depend on its rank. The majority voting by gene scores across samples is used to select top highly expressed genes. The genes having average score higher than the $\beta$ parameter (default $\beta=0.5$ ) will be selected. Top highly expressed genes are then mapped onto a network scaffold to generate a set of sub-networks. The paired $t$-test is applied on those sub-networks to select only sub-networks differentially behaved between phenotypes. Finally, permutation test by randomly swapping class label is applied to test for the significance of the sub-networks. PFSNet is available for implementation in R programming. ${ }^{22}$ Here, PFSNet is used as a baseline method, which can be incorporated in the next release of GAT. For the parameters setting, as there may be some limitation of the tool that we have not found yet, by using the default parameters set, it cannot identify the disease sub-network in most datasets except for Colorectal1 datasets. So, this study adjusts the two threshold parameters of PFSNet (t1 and $\mathrm{t} 2$ ) and found that setting these thresholds as 0.7 and 0.6, respectively, can generate a decent number of sub-networks.

\subsection{Classification parameter adjustment}

In practice, the classification performance of each classifier depends significantly on parameterization. ${ }^{44}$ For SVM, the kernel functions and the C-penalty may be adjusted for different classification problems. ${ }^{45}$ The learning rate and the momentum are the important parameters that enable MLP to escape the local maxima. However, setting these parameters too high may result in the missing of global maxima. ${ }^{46}$ In $\mathrm{RF}$, the number of trees and the size of variable subspace are the key factors enabling the classifier to be tolerant to the over-fitting problem. ${ }^{47}$

The underlying classification parameters were adjusted in this study using a sequential search with $5 \times 2$ fold cross-validation. The $5 \times 2$ fold cross-validation divides the datasets into two equal subsets and then uses one set as the training set 
Table 2. Ranges of parameters used in this study.

\begin{tabular}{lc}
\hline Parameter & Range of value \\
\hline SVM Kernel & Linear, Polynomial, Radial basis function, Sigmoid \\
SVM c-penalty & $1-5$ (by 1$)$ \\
MLP learning rate & $0-0.5$ (by 0.1$)$ \\
MLP momentum & $0-0.5$ (by 0.1$)$ \\
RF number of trees & $200-1000$ (by 200) \\
RF mtry & $\log (m)-2, \log (m)-1, \log (m), \log (m)+1, \log (m)+2$ \\
& where $m$ is the number of features \\
\hline
\end{tabular}

and the other set as the test set, and vice versa. This cross-validation process is repeated five times to provide a statistical estimate of the model ${ }^{48}$ In this study, we prefer $5 \times 2$ fold cross-validation to the traditional $k$-fold cross-validation because of the relatively small size of some datasets used. Also $5 \times 2$ fold cross-validation has been found to be a more powerful method for comparing classifiers and optimizing the parameters. ${ }^{49}$ The optimal parameter set is the set that gives the highest AUC. If there is more than one resulting set yielding the highest AUC, the highest recall will be used to break the tie. Then, the optimal parameter set of each classification and each disease will be validated by using an independent test set of the same disease. The varying parameters used in this study are shown in Table 2.

\subsection{Evaluation by gene-set level and gene level agreement}

Besides the AUC and recall, another way to assess the robustness of gene expression analysis approach is to find the agreement of the markers identified from two datasets. In this study, Jaccard-like agreement was used to calculate the withindataset and between-dataset agreements of both gene-set level and gene level as shown in Eqs. (4) and (5), respectively.

$$
\text { Gene-set Level Agreement }=\frac{\left|G S_{1} \cap G S_{2}\right|}{\left|G S_{1} \cup G S_{2}\right|},
$$

where $G S_{1}$ and $G S_{2}$ are sets of gene-sets identified in first and second datasets, respectively. $\left|G S_{X}\right|$ is size or number of gene-sets in set $G S_{X}$.

$$
\text { Gene Level Agreement }=\frac{\left|G_{1} \cap G_{2}\right|}{\left|G_{1} \cup G_{2}\right|},
$$

where $G_{1}$ and $G_{2}$ are sets of genes from gene-sets identified in the first and second datasets, respectively, and $\left|G_{X}\right|$ is the size or number of genes in set $G_{X}$. The withindataset agreement is the agreement between markers identified from sub-dataset and whole dataset. In this part of the study, the whole dataset is divided into three subdatasets, each of which has no-overlapping members with the others. Then, the markers are identified and compared with the markers set identified by using the whole dataset. The within-dataset agreement is calculated by averaging those 
agreements. Like cross-dataset validation, the between-dataset agreement is the agreement between two independent datasets of the same disease study.

\subsection{Performance comparison}

Since PFSNet is only applicable for binary class problems, so only four datasets namely, Breast1, Breast2, Colorectal1 and Colorectal2 datasets are used for the comparison. Multi-class lung cancer datasets (MCLung1, MCLung2) are also used to assess the performance and robustness of the GNFS and AFS methods in multi-class classification.

The cross-dataset validation results are used to compare the classification performance of those different gene subsets identified by GNFS, PFSNet and AFS. Table 3 presents a comparison of the classification performance measured by using AUC and recall. It can be seen that there is no significant difference in the classification performance among the three gene-set analysis methods. On average, AFS and GNFS seem to perform slightly better than PFSNet in both AUC and recall. Besides the classification performance, PurityB shows that these datasets have quite low compatibility levels, with values of $0.488,0.478$ and 0.484 for Colorectal, Breast and MCLung validations, respectively.

Table 4 shows the within-dataset gene-set level and gene level agreements of each dataset. PFSNet outperforms the others on this comparison. GNFS has similar performance with PFSNet ( $p$-value $=0.728$ on gene-set level agreement and $p$-value $=0.467$ on gene level agreement). PFSNet is slightly better than AFS in gene level agreement $(p$-value $=0.06)$, while in terms of gene-set level agreement, both performed similarly $(p$-value $=0.247)$.

Besides the within-dataset agreement, between-dataset agreement is much more significant, as the disease markers identified from two independent datasets are expected to share common characteristics. Table 5 presents a comparison of between-dataset agreements for gene-set level and gene level. Unlike the within-dataset agreement result, GNFS outperforms the others in gene-set level agreement for all

Table 3. Classification performance (AUC and recall) of cross-dataset validation results.

\begin{tabular}{llllllll}
\hline & \multicolumn{3}{c}{ AUC } & & \multicolumn{3}{c}{ Recall } \\
\cline { 2 - 3 } \cline { 7 - 8 } CV & AFS & GNFS & PFSNet & & AFS & GNFS & PFSNet \\
\hline Colorectal1-2 & 0.418 & 0.45 & $\mathbf{0 . 6 6 6}$ & & 0.774 & 0.774 & $\mathbf{0 . 9 3 8}$ \\
Colorectal2-1 & 0.579 & $\mathbf{0 . 7 4 1}$ & 0.556 & & 0.583 & 0.583 & $\mathbf{0 . 6 6 7}$ \\
Breast1-2 & $\mathbf{0 . 9 8 1}$ & 0.757 & 0.742 & $\mathbf{0 . 9 0 7}$ & 0.651 & 0.535 \\
Breast2-1 & 0.769 & 0.794 & $\mathbf{0 . 8 0 5}$ & & 0.889 & 0.889 & $\mathbf{1}$ \\
MCLung1-2 & $\mathbf{0 . 8 5}$ & 0.797 & - & $\mathbf{0 . 9 5 1}$ & 0.854 & - \\
MCLung2-1 & $\mathbf{0 . 8 8}$ & $\mathbf{0 . 8 8}$ & - & $\mathbf{0 . 5 2 9}$ & $\mathbf{0 . 5 2 9}$ & - \\
Average & $\mathbf{0 . 7 3 2}$ & $\mathbf{0 . 7 3 2}$ & 0.665 & & 0.733 & $\mathbf{0 . 7 3 6}$ & 0.726 \\
\hline
\end{tabular}

* The highest classification performance is highlighted in bold. 
Table 4. Within-dataset gene-set level and gene level agreements of the demonstration.

\begin{tabular}{lrrrrrrr}
\hline & \multicolumn{2}{c}{ Gene-set level agreement (\%) } & & \multicolumn{2}{c}{ Gene level agreement (\%) } \\
\cline { 2 - 3 } \cline { 7 - 8 } Dataset & AFS & GNFS & PFSNet & & AFS & GNFS & PFSNet \\
\hline Colorectal1 & 8.81 & 27.14 & $\mathbf{3 0 . 6 8}$ & & 7.7 & 10.78 & $\mathbf{2 3 . 4 4}$ \\
Colorectal2 & 22.64 & 36.37 & $\mathbf{5 5 . 0 9}$ & & 29.46 & 22.26 & $\mathbf{4 9 . 1 2}$ \\
Breast1 & 27.02 & $\mathbf{2 9 . 7 1}$ & 13.15 & & 16.21 & $\mathbf{3 7 . 2 6}$ & 16.03 \\
Breast2 & 19.26 & 30.49 & $\mathbf{3 5 . 9 1}$ & & 21.59 & 21.16 & $\mathbf{3 7 . 4}$ \\
MCLung1 & 9.87 & $\mathbf{5 2 . 3 4}$ & - & & 12.15 & $\mathbf{2 7 . 2 4}$ & - \\
MCLung2 & 26.08 & $\mathbf{5 9 . 5 2}$ & - & & $\mathbf{2 3 . 1 4}$ & 22.01 & - \\
\hline
\end{tabular}

* The highest agreement level is highlighted in bold.

Table 5. Between-dataset gene-set level and gene level agreements of the demonstration.

\begin{tabular}{lrrrrrrr}
\hline & \multicolumn{2}{c}{ Gene-set level agreement (\%) } & & \multicolumn{2}{c}{ Gene level agreement (\%) } \\
\cline { 2 - 3 } Dataset & AFS & GNFS & PFSNet & & AFS & GNFS & PFSNet \\
\hline Colorectal & 7.27 & $\mathbf{2 6 . 3 2}$ & 21.59 & & 3.81 & 7.24 & $\mathbf{1 2 . 4 1}$ \\
Breast & 10.11 & 7.69 & $\mathbf{1 5}$ & & 3.59 & $\mathbf{5 . 1 3}$ & 3.28 \\
MCLung & 15.79 & $\mathbf{4 3 . 7 5}$ & & & $\mathbf{9 . 6 6}$ & 7.69 & \\
\hline
\end{tabular}

*The highest agreement level is highlighted in bold.

cases except breast datasets, in which GNFS achieved similar gene level agreement with PFSNet.

To biologically interpret the result, the markers identified by GNFS, PFSNet and AFS of each dataset are mapped to KEGG pathways ${ }^{29}$ via KEGG mapper. Table 6 shows the top three mapped pathways for each dataset based on the number of genes found in each pathway.

The list of mapped pathways shows that the gene markers identified by different methods are found to be from the common pathways across datasets of the same disease. Also, all pathways presented in Table 6 are found to be cancer related pathways, especially "Pathways in cancer". For HTLV-I or Human T-lymphotropic virus type-I Infection pathway is found to associate with the development of colorectal, liver and lung cancers. ${ }^{50}$ For MAPK pathway, Rap1 and PI3K-Akt signaling pathways are involved with multiple biological processes, including cell adhesion, cell migration, cell cycle, etc. Rap1 has been found to a play role in cancer tumorigenesis and cancer metastasis while MAPK and PI3K-Akt components have been found to be altered in cancers. ${ }^{51-53}$ For the remaining pathways listed in Table 6 , the relationships between them and cancer development are also supported by evidence from multiple research projects. ${ }^{54-64}$

In PFSNet result, large number of genes is selected as candidates for disease markers in the Colorecltall dataset. Further steps of disease marker identification can be performed to filter the candidates by using this list of genes as prior knowledge 
Table 6. Mapped pathways for the gene markers of each dataset.

\begin{tabular}{|c|c|c|c|}
\hline Dataset & GNFS & PFSNet & AFS \\
\hline Colorectal1 & $\begin{array}{l}\text { - HTLV-I Infection } \\
\text { - Pathways in cancer } \\
\text { - Rap1 signaling pathway }\end{array}$ & $\begin{array}{l}\text { - Pathways in cancer } \\
\text { - Regulation of actin } \\
\text { cytoskeleton } \\
\text { - Purine metabolism }\end{array}$ & $\begin{array}{l}\text { - Pathways in cancer } \\
\text { - Proteoglycans in cancer } \\
\text { - PI3K-Akt signaling pathway }\end{array}$ \\
\hline Colorectal2 & $\begin{array}{l}\text { - Pathways in cancer } \\
\text { - HTLV-I Infection } \\
\text { - MAPK signaling pathway }\end{array}$ & $\begin{array}{l}\text { - Purine metabolism } \\
\text { - Pathways in cancer } \\
\text { - PI3K-Akt signaling } \\
\text { pathway }\end{array}$ & $\begin{array}{l}\text { - Pathways in cancer } \\
\text { - HTLV-I Infection } \\
\text { - PI3K-Akt signaling pathway }\end{array}$ \\
\hline Breast1 & $\begin{array}{l}\text { - Pathways in cancer } \\
\text { - Rap1 signaling pathway } \\
\text { - PI3K-Akt signaling pathway }\end{array}$ & $\begin{array}{l}\text { - Pathways in cancer } \\
\text { - Regulation of actin } \\
\text { cytoskeleton } \\
\text { - PI3K-Akt signaling } \\
\text { pathway }\end{array}$ & $\begin{array}{l}\text { - Pathways in cancer } \\
\text { - PI3K-Akt signaling pathway } \\
\text { - Proteoglycans in cancer }\end{array}$ \\
\hline Breast2 & $\begin{array}{l}\text { - Pathways in cancer } \\
\text { - Rap1 signaling pathway } \\
\text { - PI3K-Akt signaling pathway }\end{array}$ & $\begin{array}{l}\text { - Pathways in cancer } \\
\text { - PI3K-Akt signaling } \\
\text { pathway } \\
\text { - Regulation of actin } \\
\text { cytoskeleton }\end{array}$ & $\begin{array}{l}\text { - PI3K-Akt signaling pathway } \\
\text { - Pathways in cancer } \\
\text { - Focal adhesion }\end{array}$ \\
\hline MCLung1 & $\begin{array}{l}\text { - Rap1 signaling pathway } \\
\text { - Pathways in cancer } \\
\text { - Regulation of actin sytoskeleton }\end{array}$ & - & $\begin{array}{l}\text { - Pathways in cancer } \\
\text { - Proteoglycans in cancer } \\
\text { - PI3K-Akt signaling pathway }\end{array}$ \\
\hline MCLung2 & $\begin{array}{l}\text { - Pathways in cancer } \\
\text { - HTLV-I Infection } \\
\text { - Rap1 signaling pathway }\end{array}$ & - & $\begin{array}{l}\text { - Pathways in cancer } \\
\text { - HTLV-I Infection } \\
\text { - PI3K-Akt signaling pathway }\end{array}$ \\
\hline
\end{tabular}

for PinnacleZ. The result shows 13 sub-network biomarkers are found. Among these, sub-networks with at least 5 gene members are selected as markers. A visualization is provided in GAT as shown in Fig. 2. By inspecting the markers against colorectal cancer related genes reported by HugeNavigator, ${ }^{65}$ the results show that 7 out of 20 genes have been reported to be related to colorectal cancer.

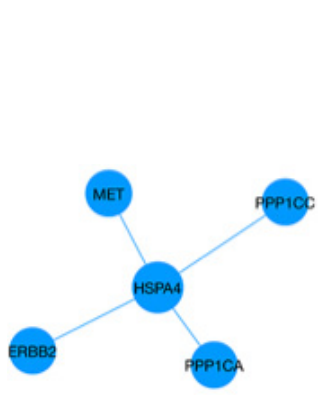

(a)

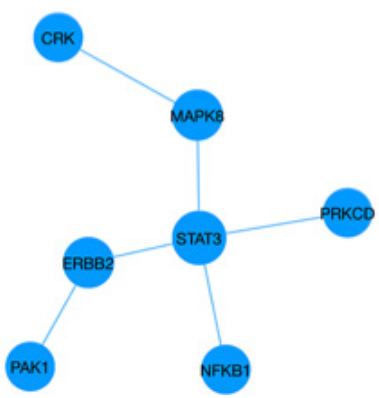

(b)

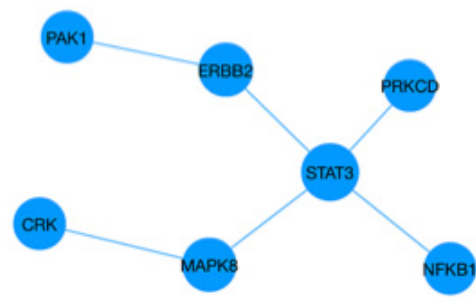

(c)

Fig. 2. Six sub-networks identified by PinnacleZ from candidate genes selected by PFSNet from Colorectal1 dataset. 


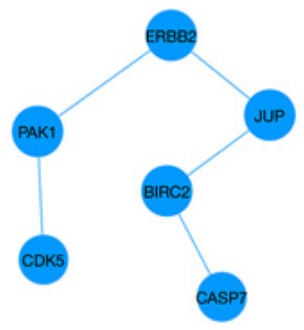

(d)

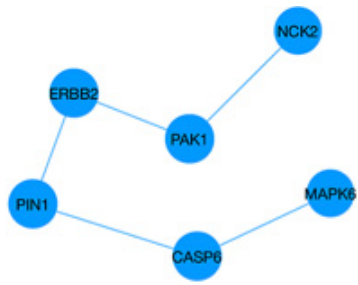

(e)

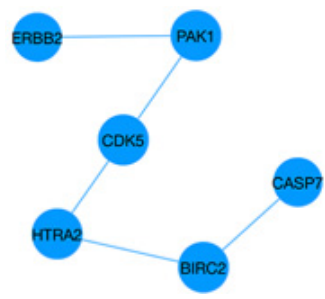

(f)

Fig. 2. (Continued)

\section{Discussion}

This work proposes GAT as a comprehensive tool for biomarker identification and disease classification. Several gene-set-based (i.e. CORGs, NCFS- $i$ and AFS) and network-based (i.e. GNFS) gene expression analysis approaches have been implemented on the current version of GAT. In addition, significance of biomarkers identified by these methods can be assessed by $k$-fold cross-validation and/or crossdataset validation with this tool. Several other features including functional analysis and network visualization are also available to help a user to perform insightful analysis of the resulting networks and genes. Most importantly, GAT is a web-based tool that enables a user to perform advanced analysis without the need of computer programming or a high-performance computing unit.

In the tool demonstration, we compare two available methods in GAT (AFS and GNFS) with the recently proposed method, PFSNet, which has been reported to outperform other common approaches. Considering the gene-set level and gene level agreement results (Table 5), the network-based analysis approaches (GNFS and PFSNet) show similar performance in finding consistent markers across datasets. Both outperform the AFS method, which does not make use of the gene network data. This is not surprising because the incorporation of additional biological information, e.g. network data would generally lead to a lower false positive rate. Although the compatibility levels between datasets and the agreement level of the gene markers between the datasets are not high (Table 5), the biological interpretation using the KEGG mapper indicates that those gene markers are primarily found in the same modules or pathways related to cancer development. This suggests the potential of the tool in extracting gene markers from common disease functional modules. Additionally, applying further analysis of PinnacleZ and network visualization can help to select those gene markers more precisely.

Even though the network-based approaches can identify consistent biomarkers, it is still difficult to accurately classify cancer samples using only gene expression data due to the heterogeneity of cancer samples. In each cancer, multiple subtypes can be classified and the markers of one subtype cannot be applied to the others. As a result, the model of heterogeneous cancer tends to have poor performance. This 


\section{W. Engchuan et al.}

heterogeneity can be observed in the within dataset agreement results. As shown in the within dataset analysis, several factors are controlled and the data of those samples are highly compatible with each other. However, the result shows that within dataset agreements are low in some datasets, e.g. gene-level agreement of Colorectal1 (AFS and GNFS) and gene-level agreement of Breast1 (AFS and PFSNet). So, to address the heterogeneity in the analysis, more information or data need to be integrated in addition to gene expression data. Many approaches have been developed to integrate multiple types of biological data (e.g. methylation in CpG island, copy number variations, etc.) along with gene expression analysis to fulfill the understanding of this complex disease. ${ }^{66,67}$ With the integration of methylation profiles and CNV data, the noises in gene expression data can be elaborated. In future work, more layers of biological data may be included to further improve the gene-set-based method. Additionally, in the analysis of microarray data across different experiments, the experimental variations or "batch effects" may also confound the analysis. ${ }^{25,68}$ Several factors can induce changes in expression levels differently. If different procedures are applied in different batches, the expression levels measured from samples of the same phenotype will be different. Thus, the expression data of one sample cannot represent the other of the same disease. To remove these uncontrollable effects, the standard batch effects correction methods should be implemented to adjust the data in a future version of GAT.

\section{Conclusions}

This study demonstrates a gene-set-based microarray data analysis tool using the newly developed GAT on six microarray datasets. With the currently available approaches in GAT, we found that the classification performance of cross-dataset validation can still be improved in order to have a reliable disease diagnostic model. Incidentally, the cross-dataset validation results can be used to suggest reasonable models that can be applied for the actual disease diagnosis of the three cancers. In the demonstration, the two methods offered in GAT, namely AFS and GNFS, are compared with an existing tool namely PFSNet. The consistency results show that GNFS performance is as good as PFSNet and outperforms AFS, which does not use gene network data. In the future, we shall consider implementing PFSNet as part of GAT.

The proposed GAT framework provides an online resource to analyze microarray data by integrating several gene-set analysis approaches. The gene-set activity tool and the transformed gene-set activity data used in this study are publicly provided at http://gat.sit.kmutt.ac.th.

\subsection{Future work}

In addition to currently available features, we plan to implement several features to render GAT a complete gene-set analysis environment in a future release. In the data 
pre-processing side, missing data imputation and batch effects correction will be added to the toolbox. Hence, users can perform cross-platform studies, with different datasets covering different numbers of genes. ${ }^{24}$ Additionally, the batch effects altering gene expression levels can be corrected. The current online GAT does not provide parameters adjustment of the model due to limited server resource allocation. However, this limitation is being addressed and will be covered in a future release. Besides the provided gene-set analysis methods, users could develop their own algorithm(s) and incorporate them into GAT. This feature helps promote their algorithms and the performance can be compared with the existing algorithms from within the proposed environment.

\section{Acknowledgments}

Engchuan would like to thank the National Center for Genetic Engineering and Biotechnology (BIOTEC), Thailand for funding of his completed Ph.D study. Also, this work is supported by a 2014 Office of Higher Education Grant (Wor-1) that is administered by King Mongkut's University of Technology Thonburi and also the Higher Education Research Promotion and National Research University (NRU) Project, Thailand's Office of the Higher Education Commission. Tongsima was supported in part by the Thailand Research Fund, grant number RSA58-80061. Furthermore, the authors would like to thank Ms. Katlin Kreamer-Tonin for proofreading of the final version of this paper.

\section{References}

1. Jemal A, Bray F, Center MM, Ferlay J, Ward E, Forman D, Global cancer statistics, $C A$ : A Cancer J Clin 61:69-90, 2011.

2. Rosemary Y, Population aging and cancer: A cross-national concern, Cancer J, 11:437$441,2005$.

3. Lakhani S, Ashworth A, Microarray and histopathological analysis of tumours: The future and the past? Nat Rev Cancer 1:151-157, 2001.

4. Bild AH, Yao G, Chang JT, Wang Q, Potti A, Chasse D, Joshi MB, Harpole D, Lancaster JM, Berchuck A, Olson JA, Marks JR, Dressman HK, West M, Nevin JR, Oncogenic pathway signatures in human cancers as a guide to targeted therapies, Nature 439:353$357,2006$.

5. Su J, Yoon B-J, Dougherty ER, Accurate and reliable cancer classification based on probabilistic inference of pathway activity, PLoS One 4:e8161, 2009.

6. Su AI, Welsh JB, Sapinoso LM, Kern SG, Dimitrov P, Lapp H, Schultz PG, Powell SM, Moskaluk CA, Frierson HF Jr, Hampton GM, Molecular classification of human carcinomas by use of gene expression signatures, Cancer Res 61:7388-7393, 2001.

7. Wang Y, Klijn JG, Zhang Y, Sieuwerts AM, Look MP, Yang F, Talantov D, Timmermans M, Meijer-van Gelder ME, Yu J, Jatkoe T, Berns EMJJ, Atkins D, Foekens JA, Gene-expression profiles to predict distant metastasis of lymph-node-negative primary breast cancer, Lancet 365:671-679, 2005.

8. Dupuy A, Simon RM, Critical review of published miroarray studies for cancer outcome and guidelines on statistical analysis and reporting, J Natl Cancer Inst 9:147-157, 2007. 
9. Lu Y, Han J, Cancer classification using gene expression data, Inf Syst 28:243-268, 2008.

10. Michiels S, Koscielny S, Hill C, Prediction of cancer outcome with microarrays a multiple random validation strategy, Lancet 365:488-492, 2005.

11. Ein-Dor L, Suk O, Domany E, Thousands of samples are needed to generate a robust gene list for predicting outcome in cancer, Proc Natl Acad Sci USA 103:5923-5928, 2006.

12. Vogelstein B, Kinzler KW, Cancer genes and the pathways they control, Nat Med 10:789-799, 2004.

13. Distaso A, Abatangelo L, Maglietta R, Creanza TM, Piepoli A, Carella M, D'Addabbo A, Ancona N, Biological and functional analysis of statistically significant pathways deregulated in colon cancer by using gene expression profiles, Int J Biol Sci 4:368-378, 2008.

14. Miecznikowski JC, Wang D, Liu S, Sucheston L, Gold D, Comparative survival analysis of breast cancer microarray studies identifies important prognostic genetic pathways, BMC Cancer 10:573, 2010.

15. Subramanian A, Tamayo P, Mootha VK, Mukherjee S, Ebert BL, Gillette MA, Paulovich A, Pomeroy SL, Golub TR, Lander ES, Mesirov JP: Gene set enrichment analysis, a knowledge-based approached for interpreting genome-wide expression profiles, PNAS 102:15545-15550, 2005.

16. Guo Z, Zhang T, Li Z, Wang Q, Xu J, Yu H, Zhu J, Wang H, Wang C, Topol EJ, Wang $\mathrm{Q}$, Rao S, Toward precise classification of cancers based on robust gene functional expression profiles, BMC Bioinf 6:58, 2005.

17. Lee E, Chuang HY, Kim JW, Ideker T, Lee D, Inferring pathway activity toward precise disease classification, PLoS Comput Biol 4:e1000217, 2008.

18. Chan JH, Sootanan P, Larpeampaisarl P, Feature selection of pathway markers for microarray-based disease classification using negatively correlated feature sets, Int Joint Conf Neural Networks (IJCNN) IEEE Press, New York, pp. 3293-3299, 2011.

19. Sootanan P, Prom-on S, Meechai A, Chan JH, Pathway-based microarray analysis for robust disease classification, Neural Comput Appl 21:649-660, 2011.

20. Engchuan W, Chan JH, Pathway activity transformation for multi-class classification of lung cancer datasets, Neurocomputing 165:81-89, 2015.

21. Doungpan N, Engchuan W, Meechai A, Chan JH, GNFS: A Network-based approach for microarray-based cancer classification (unpublished preprint).

22. Lim K, Wong L, Finding consistent disease sub-networks using PFSNet, Bioinformatics 30:189-196, 2014.

23. American Cancer Society, Cancer Facts 83 Figures 2014, American Cancer Society, Atlanta, 2014.

24. Engchuan W, Meechai A, Tongsima S, Chan JH, Handling batch effects on cross-platform classification of microarray data, Intl $J$ Adv Intell Paradigms 8(1):59-76, 2016.

25. Johnson WE, Li C, Rabinovic A, Adjusting batch effects in microarray expression data using empirical Bayes methods, Biostatistic 8:118-127, 2007.

26. Edgar R, Domrachev M, Lash AE, Gene expression omnibus: NCBI gene expression and hybridization array data repository, Nucleic Acids Res 30:207-229, 2002.

27. Soh D, Dong D, Guo Y, Wong L, Consistency, comprehensiveness, and compatibility of pathway databases, BMC Bioinf 11:449, 2010.

28. Hall M, Frank E, Holmes G, Pfahringer B, Reutemann P, Witten IH, The WEKA data mining software: An update, SIGKDD Explorations 11:10-18, 2009.

29. Kanehisa M, Araki M, Goto S, Hattori M, Hirakawa M, Itoh M, Katayama T, Kawashima S, Okuda S, Tokimatsu T, Yamanishi Y, KEGG for linking genomes to life and the environment, Nucleic Acids Res 36:D480-D484, 2008. 
30. Turashvili G, Bouchal J, Baumforth K, Wei W, Dziechciarkova M, Ehrmann J, Klein J, Fridman E, Skarda J, Srovnal J, Hajduch M, Murray P, Kolar Z, Novel markers for differentiation of lobular and ductal invasive breast carcinomas by laser microdissection and microarray analysis, BMC Cancer 7:55, 2007.

31. Richardson AL, Wang ZC, De Nicolo A, Lu X, Brown M, Miron A, Liao X, Iglehart JD, Livingston DM, Ganesan S, X chromosomal abnormalities in basal-like human breast cancer, Cancer Cell 9:121-132, 2006.

32. Hong Y, Ho KS, Eu KW, Cheah PY, A susceptibility gene set for early onset colorectal cancer that integrates diverse signaling pathways: Implication for tumorigenesis, Clin Cancer Res 13:1107-1114, 2007.

33. Sabates-Bellver J, Van der Flier LG, de Palo M, Cattaneo E, Maake C, Rehrauer H, Laczko E, Kurowski MA, Bujnicki JM, Menigatti M, Luz J, Ranalli TV, Gomes V, Pastorelli A, Faggiani R, Anti M, Jiricny J, Clevers H, Marra G, Transcriptome profile of human colorectal adenomas, Mol Cancer Res 5:1263-1275, 2007.

34. Tarca AL, Lauria M, Unger M, Bilal E, Boue S, Kumar Dey K, Koeppl H, Martin F, Meyer P, Nandy P, Norel R, Peitsch M, Rice JJ, Romero R, Stolovitzky G, Talikka M, Xiang Y, Zechner C, Improver DSC collarborators, strengths and limitations of microarray-based phenotype prediction: Lessons learned from the IMPROVER Diagnostic Signature Challenge, Bioinformatics 29:2892-2899, 2013.

35. Cortes C, Vapnik V, Support-vector networks, Mach Learn 20:273-297, 1995.

36. Li T, Zhang C, Ogihara M, A comparative study of feature selection and multiclass classification methods for tissue classification based on gene expression, Bioinformatics 20:2429-2437, 2004.

37. Ringnér M, Perterson C, Microarray-based cancer diagnosis with artificial neural networks, BioTechniques 34:S30-S35, 2003.

38. Díaz-Uriarte R, De Andres SA, Gene selection and classification of microarray data using random forest, BMC Bioinf 7:3, 2006.

39. Jiang P, Wu H, Wang W, Ma W, Sun X, Lu Z, MiPred: Classification of real and pseudo microRNA precursors using random forest prediction model with combined features, Nucleic Acids Res 35(Suppl 2):W339-W344, 2007.

40. Kotsiantis S, Kanellopoulos D, Pintelas P, Handling imbalanced dataset: A review, GESTS Int. Trans Com Sci Eng 30:25-36, 2006.

41. Goutte C, Gaussier E, A probabilistic interpretation of precision, recall and F-score, with implication for evaluation, Advances in Information Retrieval, Springer, Berlin Heidelberg, pp. 345-359, 2005.

42. Taminau J, Lazar C, Meganck S, Nowe A, Comparison of merging and meta-analysis as alternative approaches for integrative gene expression analysis, ISRN Bioinf, 345106, 2014 .

43. Chuang HY, Lee E, Liu YT, Lee D, Ideker T, Network-based classification of breast cancer metastasis, Mol Syst Biol 3:140, 2007.

44. Imbault F, Lebart K, A stochastic optimization approach for parameter tuning of support vector machines, in Proc 17th Int Conf Pattern Recognition (ICPR 2004), Vol. 4, pp. 597-600, 2004.

45. Cherkassky V, Ma Y, Practical selection of SVM parameters and noise estimation for SVM regression, Neural Networks 17(1):113-126, 2004.

46. Yu CC, Liu BD, A backpropagation algorithm with adaptive learning rate and momentum coefficient, in Proc 2002 Int Joint Conf Neural Networks (IJCNN'02), Vol. 2, pp. 1218-1223, 2002. 
47. Bouckaert RR, Frank E, Evaluating the replicability of significance tests for comparing learning algorithms, in Advances in Knowledge Discovery And Data Mining. Springer Berlin Heidelberg, pp. 3-12, 2004.

48. Dietterich TG, Approximate statistical tests for comparing supervised classification learning algorithms, Neural Comput 10:1895-1923, 1998.

49. Cieslak DA, Chawla NV, Start globally, optimize locally, predict globally: Improving performance on imbalanced data, Eighth IEEE Int Conf Data Mining (ICDM'08), pp. $143-152,2008$.

50. Arisawa K, Sobue T, Yoshimi I, Soda M, Shirahama S, Doi H, Katamine S, Saito H, Urata M, Human T-lymphotropic virus type-I infection, survival and cancer risk in southwestern Japan: A prospective cohort study, Cancer Causes Control 14:889-896, 2003.

51. Bailey CL, The Role of Rap1 in Cancer Metastasis and Pancretic Islet Beta Cell Function, Duke Dissertation, 2009.

52. Fresno Vara JA, Casado E, de Castro J, Cejas P, Belda-Iniesta C, Gonzalez-Baron M, PI3K/Akt signalling pathway and cancer, Cancer Treat Rev 30:193-204, 2004.

53. Fang JY, Richardson BC, The MAPK signalling pathways and colorectal cancer, Lancet Oncol 6:322-327, 2005.

54. Yamaguchi H, Condeelis J, Regulation of the actin cytoskeleton in cancer cell migration and invasion, Biochim Biophys Acta. 1773:642-652, 2007.

55. Polo S, Pece S, Di Fiore PP, Endocytosis and cancer, Curr Opin Cell Biol 16:156-161, 2004.

56. Glunde K, Jacobs MA, Bhujwalla ZM, Choline metabolism in cancer: Implications for diagnosis and therapy, Expert Rev Mol Diagn 6:821-829, 2006.

57. Segui B, Andrieu-Abadie N, Jaffrezou JP, Benoist H, Levade T, Sphigolipids as modulators of cancer cell death: Potential therapeutic targets, Biochim Biophys Acta Biomembr 1758:2104-2120, 2006.

58. Weber G, Enzymes of purine metabolism in cancer, Clin Biochem 16:57-63, 1983.

59. Rodriguez-Antona C, Ingelman-Sundberg M, Cytochrome P450 pharmacogenetics and cancer, Oncogene 25:1679-1691, 2006.

60. McLean GW, Carragher NO, Avizienyte E, Evans J, Brunton VG, Frame MC, The role of focal-adhesion kinase in cancer - A new therapeutic opportunity, Nat Rev Cancer 5:505-515, 2005.

61. Iozzo RV, Sanderson RD, Proteoglycans in cancer biology, tumour microenvironment and angiogenesis, J Cell Mol Med 15:1013-1031, 2011.

62. Martin TA, Jiang WG, Loss of tight junction barrier function and its role in cancer metastasis, Biochim Biophys Acta-Biomembr 1788:872-891, 2009.

63. Wong $\mathrm{CH}$, Goh KL, Chronic hepatitis B infection and liver cancer, Biomed Imag Interv $J$ 2:e7, 2006.

64. Ren Y, Zheng J, Yao X, Weng G, Wu L, Essential role of the cGMP/PKG signaling pathway in regulating the proliferation and survival of human renal carcinoma cells, Int $J$ Mol Med 34:1430-1438, 2014.

65. Yu W, Gwinn M, Clyne M, Yesupriya A, Khoury MJ, A navigator for human genome epidemiology, Nat Genet 40:124-125, 2008.

66. Stranger BE, Forrest MS, Dunning M, Igle CE, Beazley C, Thorne N, Dermitzakis ET, Relative impact of nucleotide and copy number variation on gene expression phenotypes, Science 315:848-853, 2007.

67. Sun Z, Asmann YW, Kalari KR, Bot B, Eckel-Passow JE, Baker TR, Carr JM, Khrebtukova I, Luo S, Zhang L, Schroth GP, Perez EA, Thompson EA, Integrated analysis of gene expression, CpG Island methylation, and gene copy number in breast cancer cells by deep sequencing, PLOS One 6:e17490, 2011. 
68. Larsen MJ, Thomassen M, Tan Q, Sorensen KP, Kruse TA, Microarray-based RNA profiling of breast cancer: Batch effect removal improves cross-platform consistency, BioMed Res Int 2014.

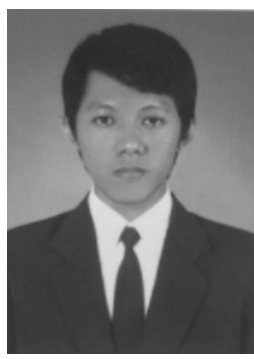

Worrawat Engchuan is a Researcher at School of Information Technology, King Mongkut's University of Technology Thonburi (KMUTT). He obtained his B.Sc. in Information Systems from Prince of Songkla University, his M.Sc. in Bioinformatics from KMUTT and his Ph.D. in Computer Science from KMUTT. He has been a sub-reviewer for a number of International Journals and reviewer for several International Conferences in the bioinformatics field. His research interests include computational systems biology, bioinformatics, and machine learning.

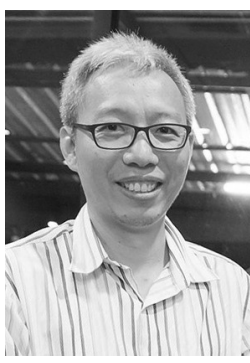

Asawin Meechai is an Assistant Professor at Department of Chemical Engineering, King Mongkut's University of Technology Thonburi (KMUTT). He also has joint faculty appointments at the Bioinformatics and Systems Biology program as well as the Biological Engineering at KMUTT. He received his B.Sc. in Biology from Chiangmai University, his M.Sc. in Biochemistry from Mahidol University and his Ph.D. in Biochemical Engineering from University of California. His research interests include metabolic engineering, bioinformatics and systems biology.

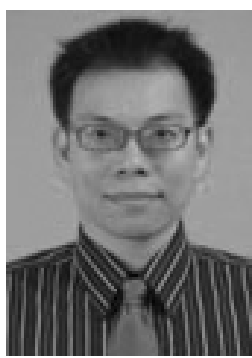

Sissades Tongsima is currently a Principal Researcher and the Head of Biostatistics and Informatics Laboratory at Genome Technology Research Unit, National Center for Genetic Engineering and Biotechnology (BIOTEC), Thailand. He received his B.Eng. in Industrial Instrumentation Engineering from King Mongkut's Institute of Technology Ladkrabang (KMIT'L) and his MSCSE and Ph.D. in Computer Science and Engineering from the University of Notre Dame, USA. His research interests include bioinformatics and computation modeling. Dr. Tongsima has published his computational biology research in many renowned journals; some of them were highlighted in the Asia-Pacific International Molecular Biology Network (AIMB-N). He has served as an executive committee of Asia Pacific Bioinformatics Network (APBioNet), and a steering member of several international consortia. He is also an associate editor of the Journal of Human Genetics (JHG), Nature Publishing Group. 


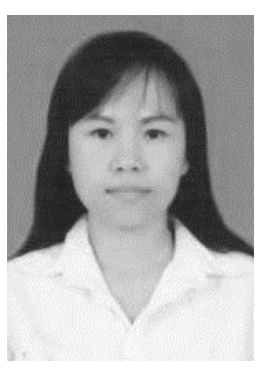

Narumol Doungpan is a Ph.D. student in Biological Engineering Program, King Mongkut's University of Technology Thonburi (KMUTT). She received her B.Sc. in Biotechnology from Mahidol University and her M.Sc. in Bioinformatics from KMUTT. Her research interests include computational systems biology and bioinformatics.

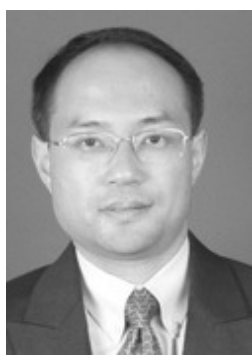

Jonathan Chan is an Associate Professor at the School of Information Technology, King Mongkut's University of Technology Thonburi (KMUTT), Thailand. He received his BASc degree in Engineering Science and his MASc and Ph.D. degrees in Chemical Engineering from the University of Toronto. He is a member of the editorial board for Neural Networks and a reviewer for a number of refereed International Journals including Information Science, Neural Computing and Applications, Memetic Computing, International Journal of Data Mining and Bioinformatics, and BMC Genomics. He has also served on the program and technical committees for numerous international conferences such as IJCNN, CEC, ICONIP, CIDM, InCoB and ISNN. Dr. Chan is a past President and current governing board member of the Asian Pacific Neural Network Assembly (APNNA), and he is a senior member of IEEE, a member of ACM, a member of International Neural Network Society (INNS) and a member of Professional Engineers of Ontario (PEO). His research interests include mathematical modeling and simulation, bioinformatics, machine learning, and parallel computing. 